\title{
Modeling of dengue outbreak prediction in Malaysia: a comparison of neural network and nonlinear regression model
}

\begin{abstract}
Malaysia has a good dengue surveillance system but there have been insufficient findings on suitable model to predict future dengue outbreak. This study aims to design a neural network model (NNM) and nonlinear regression model (NLRM) using different architectures and parameters incorporating time series, location and rainfall data to define the best architecture for early prediction of dengue outbreak. Four architecture of NNM and NLRM were developed in this study. Architecture I involved only dengue cases data, Architecture II involved combination of dengue cases data and rainfall data, Architecture III involved proximity location dengue cases data, while Architecture IV involved the combination of all criterion. The parameters studied in this research were adjusted for optimal performance. These parameters are the learning rate, momentum rate and number of neurons in the hidden layer. The performance of overall architecture was analyzed and the result shows that the MSE for all architectures by using NNM is better compared by NLRM. Furthermore, the results also indicate that architecture IV performs significantly better than other architecture in predicting dengue outbreak and it is therefore proposed as a useful approach in the problem of time series prediction of dengue outbreak.
\end{abstract}

